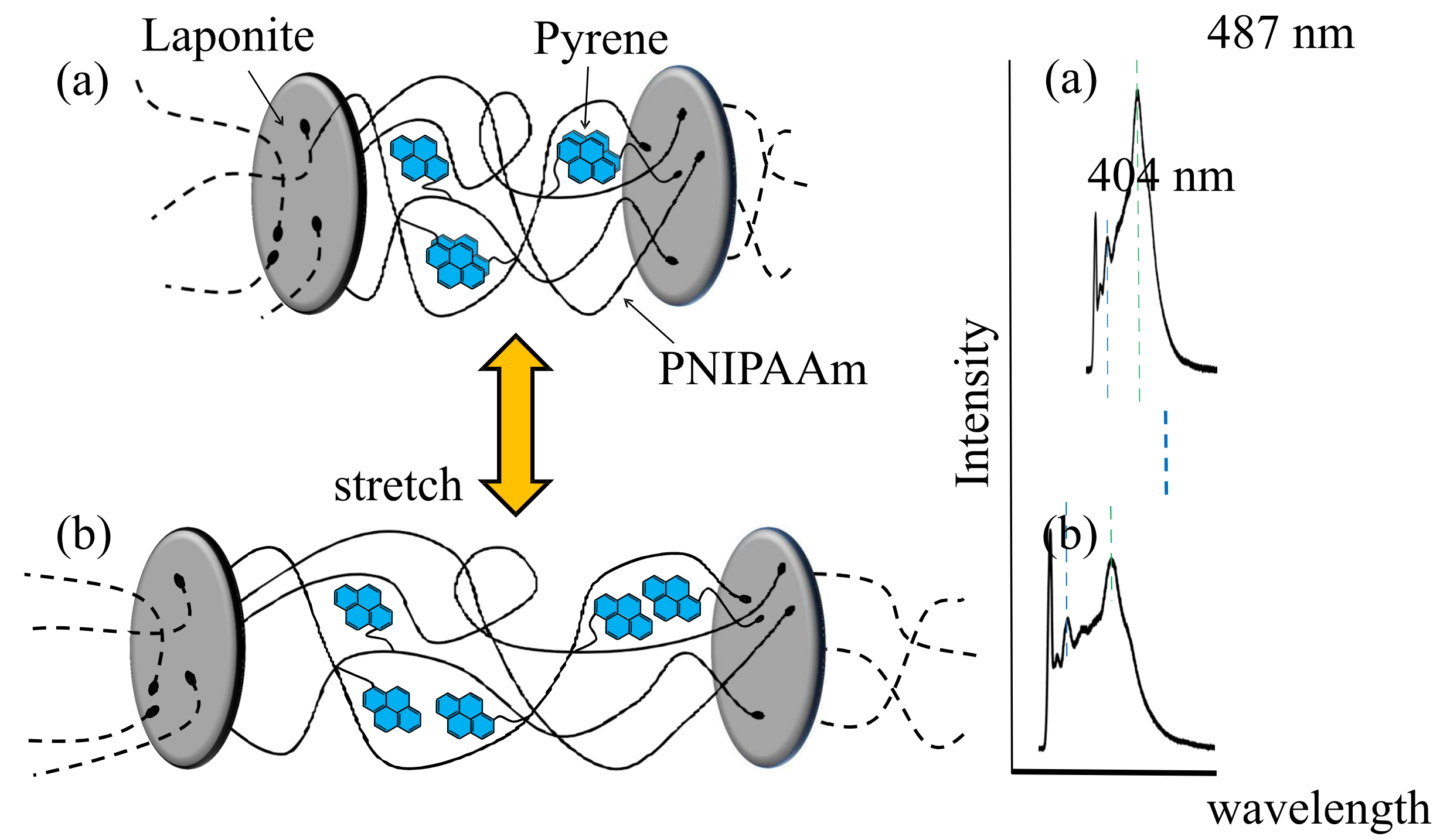




\title{
Mechanochromic response of pyrene functionalized nanocomposite hydrogels
}

\author{
Filippo Cellini ${ }^{\mathrm{a}}$, Leanne Block ${ }^{\mathrm{a}}$, Jie Li ${ }^{\mathrm{a}}$, Sachin Khapli ${ }^{\mathrm{b}}$, Sean D. Peterson ${ }^{\mathrm{a}, \mathrm{c}}$, Maurizio Porfiri ${ }^{\mathrm{a}, *}$ \\ ${ }^{a}$ Department of Mechanical and Aerospace Engineering, Tandon School of Engineering, \\ New York University, Six MetroTech Center, Brooklyn, NY 11201, USA \\ ${ }^{b}$ Engineering Division, New York University Abu Dhabi, Abu Dhabi 129188, UAE \\ ${ }^{c}$ Department of Mechanical and Mechatronics Engineering, University of Waterloo, Waterloo, Ontario N2L 3G1, Canada
}

\begin{abstract}
Mechanochromic polymers display detectable changes in their fluorescence emission upon deformation of their macromolecular structure. Here, we study reversible mechanochromism of pyrene functionalized nanocomposite hydrogels. Highly stretchable nanocomposite hydrogels are prepared by free radical polymerization of poly(N-isopropylacrylamide) and pyrene terminated poly(ethylene) glycol methacrylate copolymers in the presence of nanoclay, acting as the physical crosslinker. Mechanochromic behavior is characterized through uniaxial tensile experiments, where force, deformation, and fluorescence emission are simultaneously measured. To demonstrate the application of mechanochromic nanocomposite hydrogels in pressure sensing, membranes are used to detect differential pressures on the order of $10^{2} \mathrm{~Pa}$. We envision the integration of these mechanochromic hydrogels in the design of novel force and pressure sensors with application to mechanics, fluid dynamics, and biophysics.
\end{abstract}

Keywords: Fluorescence sensing, Monomer-excimer fluorescence, Mechanochromism, Nanocomposite hydrogel, Pressure sensing.

\section{Introduction}

Polymers can be engineered to respond to environmental stimuli through the integration of active functional groups into their structures. Different classes of active polymers have been recently proposed to selectively respond to mechanical, electrical, or chemical stimuli (Buenger et al., 2012; Debashish et al., 2010; Jo et al., 2013; Urban, 2011). Sensors based on active polymers are finding applications in life science (Amano et al., 2012; Deligkaris et al., 2010; Matzeu et al., 2015), robotics (Chen et al., 2007; Shahinpoor, 2016), and experimental mechanics and biomechanics (Ferrari et al., 2007; Shao et al., 2014).

Mechanochromic polymers are composed of a synthetic polymer embedding an active fluorescent dye molecule (Chi et al., 2012; Ciardelli et al., 2013; Haehnel et al., 2015; Pucci et al., 2011). During deformations of the polymer, detectable modifications in the fluorescence emission can be induced by controlling the aggregation and alignment of the dyes (Ciardelli et al., 2013; Haehnel et al., 2015). In particular, the transition from excimer to monomer emission due to rupture of dye aggregates (Haehnel et al., 2015; Lowe and Weder, 2002) and aggregation-induced emission phenomena (Chi et al., 2012; Li et al., 2015) are known to control the physics of fluorescence emission in mechanochromic polymers. Dyes that can afford such transitions are characterized by planar conjugated structures and by the absence of bulky side groups, structural features that facilitate the formation of supramolecular aggregates via $\pi-\pi$ stacking or hydrogen bonding (Ciardelli et al., 2013).

\footnotetext{
${ }^{*}$ Corresponding author. Tel.: +1-646-997-3681. Fax: +1-646-997-3532.

Email address: mporfiri@nyu.edu (Maurizio Porfiri)
} 
Several classes of organic dyes have been proposed for synthesis of mechanochromic polymers, such as pyrene (Roberts et al., 2012), perylene (Donati et al., 2008), stilbene (Bao et al., 2013; Cellini et al., 2014; Pucci et al., 2005), and oligo(p-phenylene vinylene) (Kunzelman et al., 2006, 2008b) derivatives. Mechanochromic response of this class of materials is often non-reversible due to the permanent flow of the polymer chains during the deformation process (Donati et al., 2008; Kunzelman et al., 2006; Pucci et al., 2005). However, recent studies have demonstrated reversible optical response in mechanochromic shape memory polymers (Kunzelman et al., 2008a) and elastomeric networks (Cellini et al., 2016; Crenshaw and Weder, 2006; Gossweiler et al., 2014; Imato et al., 2015), whereby optical properties are recovered during the inversion of the deformation process.

Nanocomposite hydrogels are a novel class of soft materials that can afford large reversible deformations (Haraguchi and Takehisa, 2002; Schexnailder and Schmidt, 2009). Nanocomposite hydrogels have received considerable attention due to their remarkable physical properties, such as high degree of mechanical toughness, elastic response over large deformations, high swelling ratios, rapid swelling-deswelling response, and optical transparency. This hydrogel class is composed of long macromolecular chains physically interconnected through nanoscale inorganic clay platelets, uniformly distributed within the polymer structure (Haraguchi, 2007). During the deformation process, clay platelets act as physical links between the chains, allowing unfolding of the polymer structure without loss of structural integrity under strains of over $1000 \%$. Different monomers have been used in the preparation of nanocomposite hydrogels, such as N-isopropylacryamide (Haraguchi et al., 2005; Haraguchi and Takehisa, 2002), N,N-dimethylacrylamide (Haraguchi et al., 2005), acrylamide (Okay and Oppermann, 2007), poly(ethylene glycol) diacrylate (Chang et al., 2010), and poly(ethylene glycol) methacrylate (Hu et al., 2010). These monomers are characterized by high solubilities in water and by the presence of polar groups that are able to effectively interact with the clay surface. Synthetic clays, such as Laponite XLG, are commonly used in the hydrogel synthesis for their ability to effectively swell and disperse in water (Haraguchi, 2007).

In this paper, we demonstrate mechanochromism of Laponite-crosslinked poly(NIPAAm-co-PEGMA) nanocomposite hydrogels fluorescently labeled with pyrene butyric acid. The synthesis of this new class of mechachromic polymers extends the approach presented in Haraguchi and Takehisa (2002) for the preparation of Laponite-crosslinked poly(NIPAAm) nanocomposite hydrogels, to enable mechachromism. The mechanism proposed to support mechanochromic response is displayed in Figure 1. The pyrene groups covalently bonded to the polymer structure undergo progressive reorganization during stretching of the network. In particular, the reorganization of pyrene aggregates in single pyrene molecules results in a detectable change of fluorescence emission. This change is manifested as an increase in the emission intensity of single molecules (monomer emission) with respect to aggregates (excimer emission). As the network recovers its

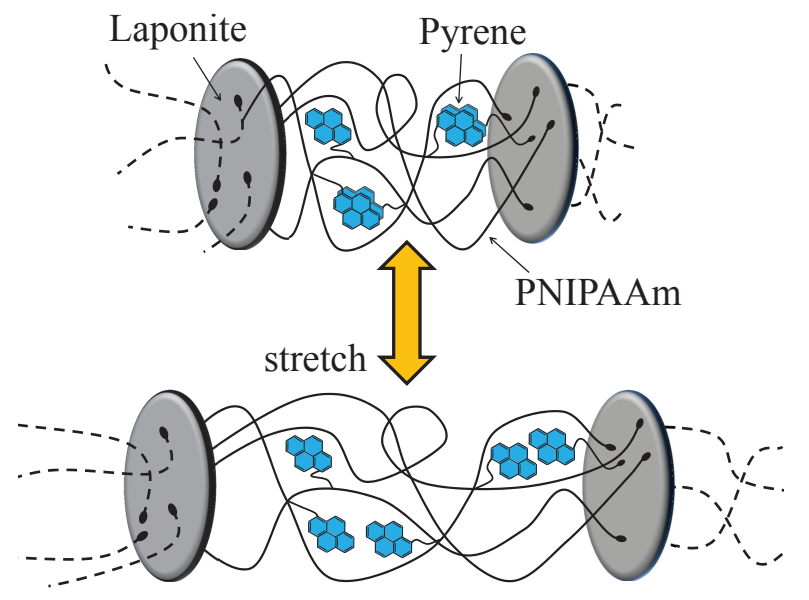

Figure 1: Mechanochromic response of pyrene-functionalized nanocomposite hydrogels, adapted from Haraguchi (2007). The stretch of the hydrogel network promotes the reorganization of pyrene molecules and reduces their interaction. 
original configuration, the process is reversed and the hydrogel returns to its original emission landscape.

We establish an experimental procedure for simultaneous acquisition of the applied force, deformation, and fluorescence emission under UV excitation, toward the systematic characterization of mechanochromic response during large deformations. To further elucidate their mechanical behavior, we consider a modified form of the Arruda-Boyce model (Boyce and Arruda, 2001) for swollen elastomeric networks, which has already been adopted for the study of PNIPAAm hydrogels (Westbrook and Qi, 2008). Similarly, we adapt the constitutive hypothesis in Cellini et al. (2016) to study mechanochromism of polyurethane elastomers and isolate the dependence of the fluorescence emission on key observables for the mechanical deformation. Finally, to demonstrate the possibility of incorporating mechanochromic nanocomposite hydrogels in mechanical sensing systems, we propose the use of hydrogel membranes to sense differential pressures on the order of a few hundred Pascals.

The rest of the paper is organized as follows. In Section 2, we describe the experimental procedures adopted for synthesis and characterization, along with the setup developed for pressure sensing. In Section 3 , we present results for optical characterization of pyrene terminated poly (ethylene glycol) methacrylate and pyrene labeled nanocomposite hydrogels and we investigate their mechanical response and mechanochromic behavior under uniaxial loading conditions and imposed pressure. The article concludes with a summary of our main findings in Section 4. In Appendix A, we report nuclear magnetic resonance spectroscopy (NMR) and mass spectrometry results for the characterization of pyrene terminated poly (ethylene glycol) methacrylate.

\section{Experimental section}

\section{Materials}

N-isorpropylacrylamide (NIPAAm, 97\%), N,N,N',N'-Tetramethylethylenediamine (TEMED, 97\%), potassium persulfate (KPS, 99\%), poly (ethylene glycol) methacrylate (PEGMA, hydroxyl-terminated; average $\mathrm{M}_{\mathrm{n}}$ 360), 1-pyrenebutyric acid (PyBA, 97\%), N,N'-dicyclohexylcarbodiimide (DCC, 99\%), 4-(dimethylamino) pyridine (DMAP, $\geq 99 \%$ ), dichloromethane (DCM, $\geq 99.9 \%$ ), and acetonitrile (ACN, $\geq 99.93 \%$ ) were purchased from Sigma Aldrich (www.sigmaaldrich.com). Laponite XLG nanoclay was kindly provided from Southern Clay Products (Rockwood Additives). Deionized water (resistivity 18.2 Mohm) was used to prepare solutions. All chemicals were used as received without further purification.

\section{Preparation of pyrene-terminated PEGMA (Py-PEGMA)}

PyBA (120 mg) was dissolved in $38 \mathrm{ml}$ DCM through sonication using a Branson 1510 Ultrasonic bath. PEGMA (1000 mg) was diluted in $2 \mathrm{ml}$ DCM and then added to the PyBA solution. In a different vial, a stoichiometric amount of DCC $(600 \mathrm{mg})$ was dissolved in $2 \mathrm{ml}$ DCM with light agitation. The DCC and PyBA-PEGMA solutions were kept at $0^{\circ} \mathrm{C}$ for 10 minutes before mixing them. A catalytic amount of DMAP $(60 \mathrm{mg})$ was added to the solution to promote the esterification reaction between the butyric acid and the hydroxyl group of the PEGMA, which is the single step reaction for the synthesis of Py-PEGMA.

The final solution was left to react for 24 hours in the dark under gentle stirring ${ }^{1}$. DCM was extracted by evaporation under low pressure at approximately $40^{\circ} \mathrm{C}$. After the complete evaporation of DCM, a dark-yellow viscous liquid was obtained. The products were redissolved in ACN. Upon addition of the solvent, sub-millimeter size crystals rapidly precipitated out of the solution forming a white deposit on the bottom of the vial. Crystals were eliminated by filtration using Whatman 1441 filter paper. The Py-PEGMA solution was collected in a glass vial and stored at room temperature. The chemical structures of unmodified PEGMA, PyBA, and Py-PEGMA are reported in Figure 2. Results for the characterization of Py-PEGMA through 1D proton nuclear magnetic resonance (NMR) spectroscopy and liquid chromatography ion trap mass spectrometry (Ion Trap LC-MS) are reported in Appendix A.

\footnotetext{
${ }^{1}$ To completely quench the reaction, residual DCC can be hydrolyzed using 1-5\% in volume of 1:1 solution of Acetic Acid: $\mathrm{H}_{2} \mathrm{O}$ and precipitates removed by filtration.
} 


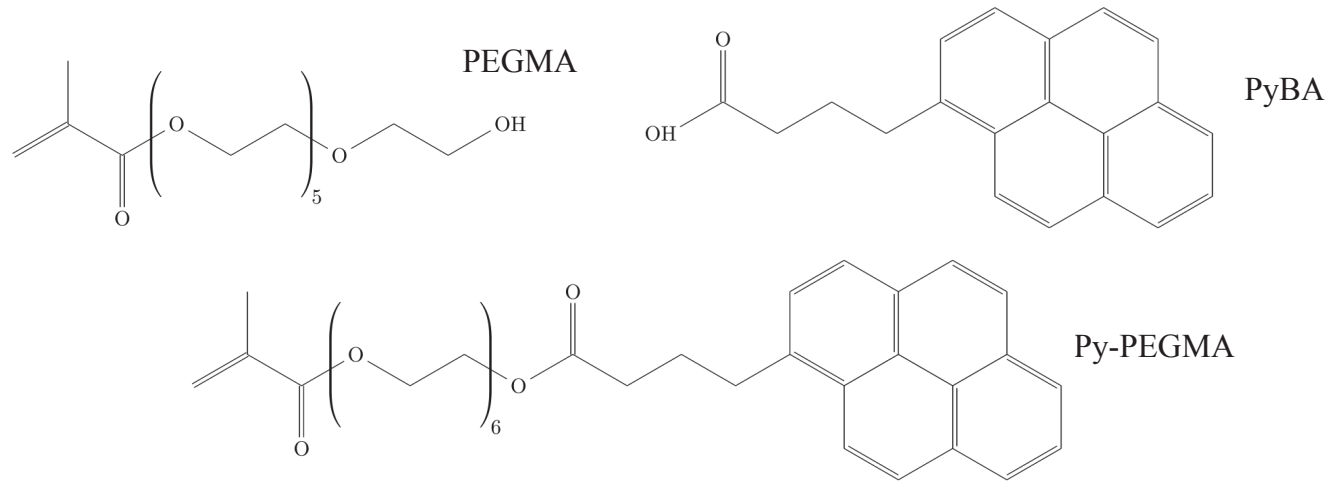

Figure 2: Chemical structures of unmodified polyethylene glycol methacrylate (PEGMA), pyrene butyric acid (PyBA), and Py-PEGMA oligomers.

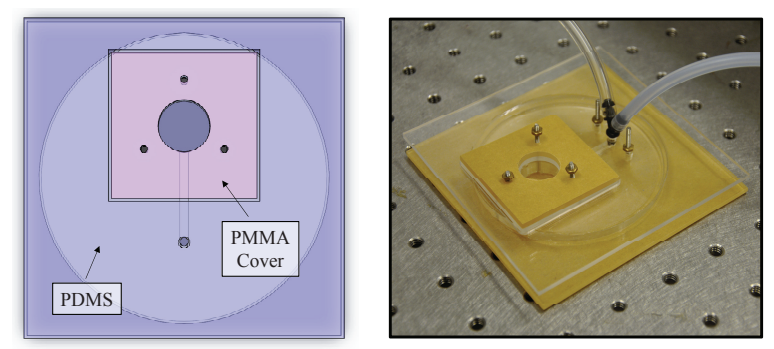

Figure 3: Experimental setup for pressure sensing.

Preparation of mechanochromic poly(NIPAAm-co-Py-PEGMA) nanocomposite hydrogels

Mechanochromic poly(NIPAAm-co-Py-PEGMA) nanocomposite hydrogels were prepared with $10 \mathrm{wt} \%$ of NIPAAm, 1.5 and $3 \mathrm{wt} \%$ of clay, and $1 \mathrm{wt} \%$ of Py-PEGMA. Weight fractions were computed with respect to the total mass of the hydrated gel (polymer + clay + solvent). Laponite XLG nanoclay was exfoliated in $8 \mathrm{ml}$ of deionized water and dispersed for 10 minutes using a Sonics VCX-500 Vibra Cell Ultrasonic Processor (47\% power level, room temperature) until a clear suspension was obtained. Monomer NIPAAm was added to the clay suspension, and high purity nitrogen was bubbled for 30 minutes through the mixture to remove oxygen.

To initiate polymerization, the reaction mixture was kept for 5 minutes at $0^{\circ} \mathrm{C}$ before the addition of $500 \mu \mathrm{l}$ of a $0.15 \mathrm{~mol}$ solution of KPS in water, $1 \mathrm{ml}$ solution of Py-PEGMA in acetonitrile, and $14 \mu \mathrm{l}$ of TEMED. The reacting mixture was then poured in a sealed mold protected from oxygen and left to polymerize for 24 hours. The nanocomposite hydrogel was extracted from the mold, weighed, and dialyzed for 24 hours in deionized water before testing.

Optical characterization of Py-PEGMA and poly(NIPAAm-co-Py-PEGMA) nanocomposite hydrogels

Fluorescence characterization of the Py-PEGMA dye-polymer conjugate in acetonitrile solution was performed by recording the emission and absorption spectra through a $3.5 \mathrm{ml}$ quartz cell. Fluorescence experiments were performed using a PTI Quanta Master 40 fluorescence spectrophotometer mounting 1200 line $/ \mathrm{mm}$ gratings for emission ( $400 \mathrm{~nm}$ blaze) and excitation ( $300 \mathrm{~nm}$ blaze), respectively. Absorption spectra were collected using a Varian Cary $50 \mathrm{UV}$-Vis spectrophotometer. A $3.5 \mathrm{ml}$ cuvette with $12.4 \mathrm{~mm}$ path length was used in the experiments.

Infrared spectra of the the Py-PEGMA were collected using a Thermo-Scientific Nicolet 6700 spectrometer mounting a Smart iTR module for attenuated total reflectance Fourier transform infrared spectroscopy (ATR-FTIR). Liquid polymer samples were dried under reduced pressure and applied on the diamond ATR crystal to measure sample transmission. To characterize the hydrogel structure and verify the polymeriza- 


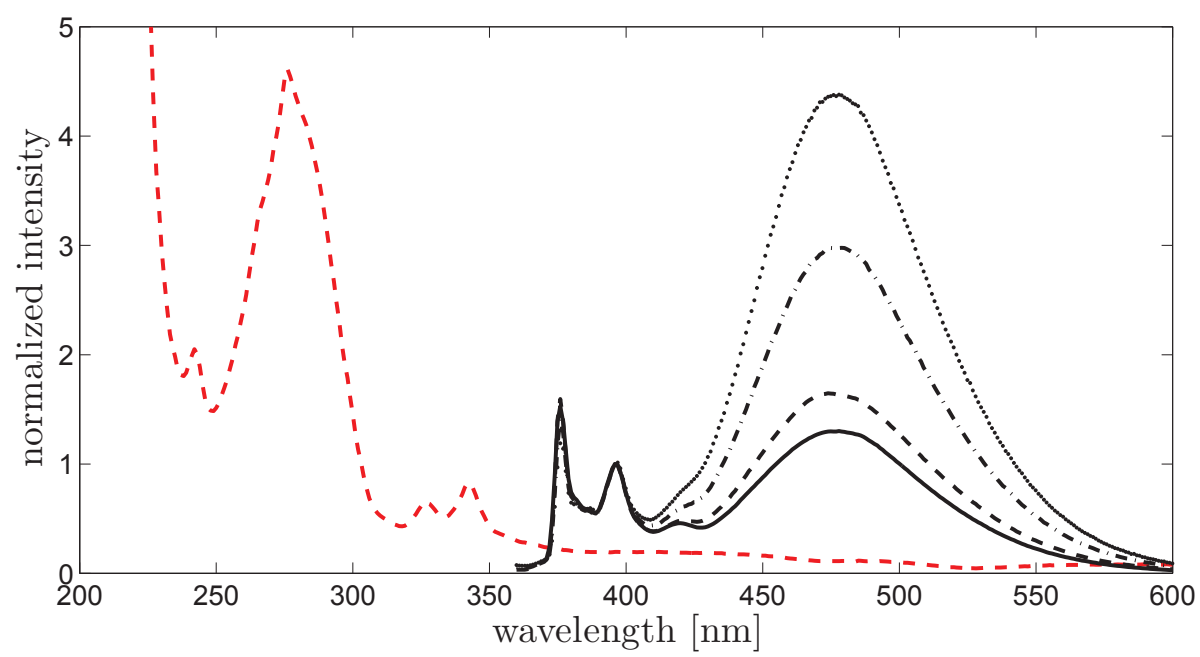

Figure 4: Fluorescence emission spectra of Py-PEGMA oligomers dissolved in acetonitrile for concentrations $1 \mathrm{mM}$ (black dotted line), $0.5 \mathrm{mM}$ (black dash-dotted line), $100 \mu \mathrm{M}$ (black dashed line), and $50 \mu \mathrm{M}$ (black solid line). Fluorescence is recorded in the $370-600 \mathrm{~nm}$ range with a $350 \mathrm{~nm}$ excitation wavelength. Spectra are normalized at $396 \mathrm{~nm}$ for comparison. The UV-Vis absorption spectrum of diluted solution ( $50 \mu \mathrm{M}$ concentration) is also presented in the $200-600 \mathrm{~nm}$ range (red-dashed line).

tion process, spectra of poly(NIPAAm-co-Py-PEGMA) nanocomposite hydrogel films were recorded using the same equipment. Before measuring the spectrum, hydrogel films were dialyzed in water to remove any unreacted monomer and thoroughly dried.

\section{Optomechanical characterization and pressure sensing}

Optomechanical characterization was performed using a setup similar to the one described in Cellini et al. (2016). Slabs of reference dimensions $42 \times 5 \times 5 \mathrm{~mm}^{3}$ were connected through a custom-made set of clamps to a Transducer Techniques $250 \mathrm{~g}$ load cell. Elongation of the slab during the test was recorded using a FLEA FL3-U3-13E4C-C camera with acquisition frequency set to 7.5 frames per second.

Fluorescence of the pyrene conjugate embedded in the hydrogel was excited using a LZ1-00UV00 high power UV light emitting diode (LED) with emission centered at $365 \mathrm{~nm}$ (www.ledengin.com). To limit overlap of the emission band of the LED with the emission of the slab, a U-340 colored glass bandpass filter (cut-off wavelength $390 \mathrm{~nm}$, www.edmundoptics.com) was positioned in front of the LED emitter. Hydrogel emission during the test was collected using an Ocean Optics USB2000+ spectrophotometer (www.oceanoptics.com) connected to a SMA air-spaced collimator (ww.thorlabs.com) through a $600 \mu \mathrm{m}$ diameter fiber optic cable with a numerical aperture of 0.22 .

Pressure sensing was demonstrated using cylindrical membranes with a diameter of $28 \mathrm{~mm}$ and a reference thickness of $2 \mathrm{~mm}$. The membranes were installed on an ad-hoc designed PDMS pneumatic circuit, see Figure 3. The circuit was connected via a Y-connector to a Sper Scientific 840099 Digital Manometer and a $12 \mathrm{~V}$ mini air-pump. Membrane inflation was recorded from one side view using a FLEA FL3-U3-13E4C-C camera with acquisition frequency set to 15 frames per second. The concurrent fluorescence emission was recorded through the same optical setup employed in the tensile experiments.

\section{Results and discussion}

Optical properties of Py-PEGMA and poly(NIPAAm-co-Py-PEGMA) nanocomposite hydrogels

Fluorescence emission of the Py-PEGMA oligomers was characterized in acetonitrile solution. Different concentrations were tested, including $1 \mathrm{mM}, 0.5 \mathrm{mM}, 100 \mu \mathrm{M}$, and $50 \mu \mathrm{M}$. Emission spectra were recorded in the $370-600 \mathrm{~nm}$ range using $350 \mathrm{~nm}$ as the excitation wavelength. Absorption spectra of the same solutions were collected in the $200-600 \mathrm{~nm}$ range. 

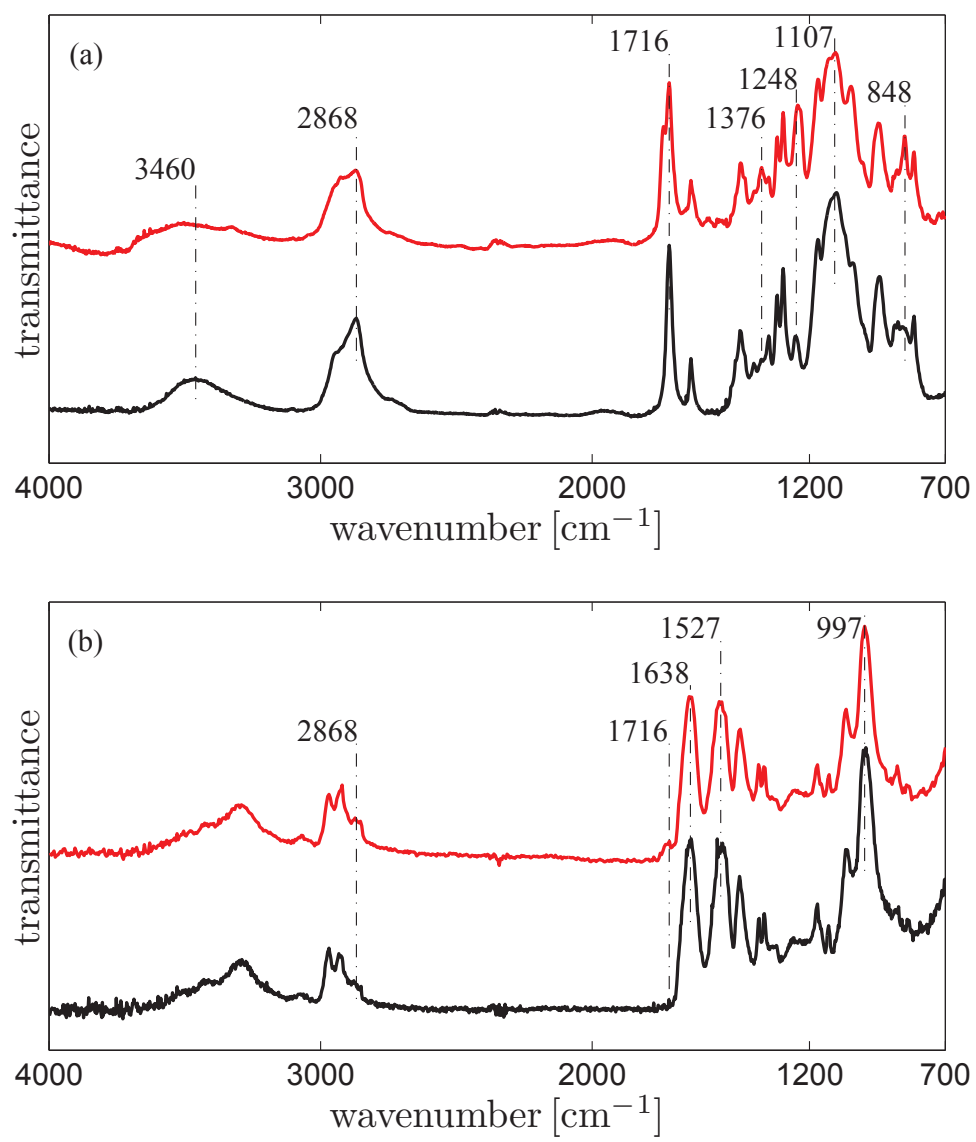

Figure 5: (a) Infrared transmission spectra of PEGMA (black line) and Py-PEGMA (red line) oligomers. (b) Infrared transmission spectra of polymerized poly(NIPAAm) (black line) and poly(NIPAAm-co-Py-PEGMA) (red line) nanocomposite hydrogels.

Figure 4 shows the fluorescence spectra of Py-PEGMA solutions; the absorption spectrum of the lowest concentration solution $(50 \mu \mathrm{M})$ is also displayed for reference. The absorption of Py-PEGMA in solution displays several distinct absorption bands, associated with different vibronic states of pyrene (Duhamel, 2012; Yao et al., 2005). Specifically, from the absorption spectrum in Figure 4, excited states of pyrene with absorption peaks at 242, 277, 327, and $342 \mathrm{~nm}$ are identifiable. A progressive red-shift of the peaks is observed with increasing Py-PEGMA concentration (spectra not shown), while the characteristic features of pyrene absorption remains substantially unchanged.

The fluorescence spectra of the polymer-dye conjugates exhibit the characteristic profile of pyrene emission, with two sharp peaks located at 376 and $396 \mathrm{~nm}$, which are associated with the first and fourth vibronic bands in the emission of single, non-interacting, Py-PEGMA molecules in solution (Bains et al., 2011; Birks and Christophorou, 1963). The wide emission band between 430 and $530 \mathrm{~nm}$ is related to the excimer emission of molecular aggregates, which are formed through self-coordination of planar pyrene structures through $\pi-\pi$ stacking. As expected, the relative emission of the aggregates with respect to single molecules increases as the concentration rises, due to the growing likelihood of interaction between pyrene molecules.

The infrared transmission spectrum of Py-PEGMA oligomers for wavenumbers ranging from 4000 to $700 \mathrm{~cm}^{-1}$ is displayed in Figure 5(a) together with the spectrum of unmodified PEGMA oligomers. Main absorption bands in PEGMA oligomers are located at: $1107 \mathrm{~cm}^{-1}$ for stretching vibrations of the $\mathrm{C}-\mathrm{O}-\mathrm{C}$ in the ether bond; 1248 and $1376 \mathrm{~cm}^{-1}$ for symmetric and antisymmetric stretching vibrations of the $\mathrm{C}-\mathrm{O}-\mathrm{C}$ in the ester bond; $1716 \mathrm{~cm}^{-1}$ for stretching vibrations of the $\mathrm{C}=\mathrm{O}$ in the ester bond; $2868 \mathrm{~cm}^{-1}$ for stretching vibrations of the $-\mathrm{CH}_{2}-$ bond; and $3460 \mathrm{~cm}^{-1}$ for stretching vibrations of the terminal hydroxyl group 

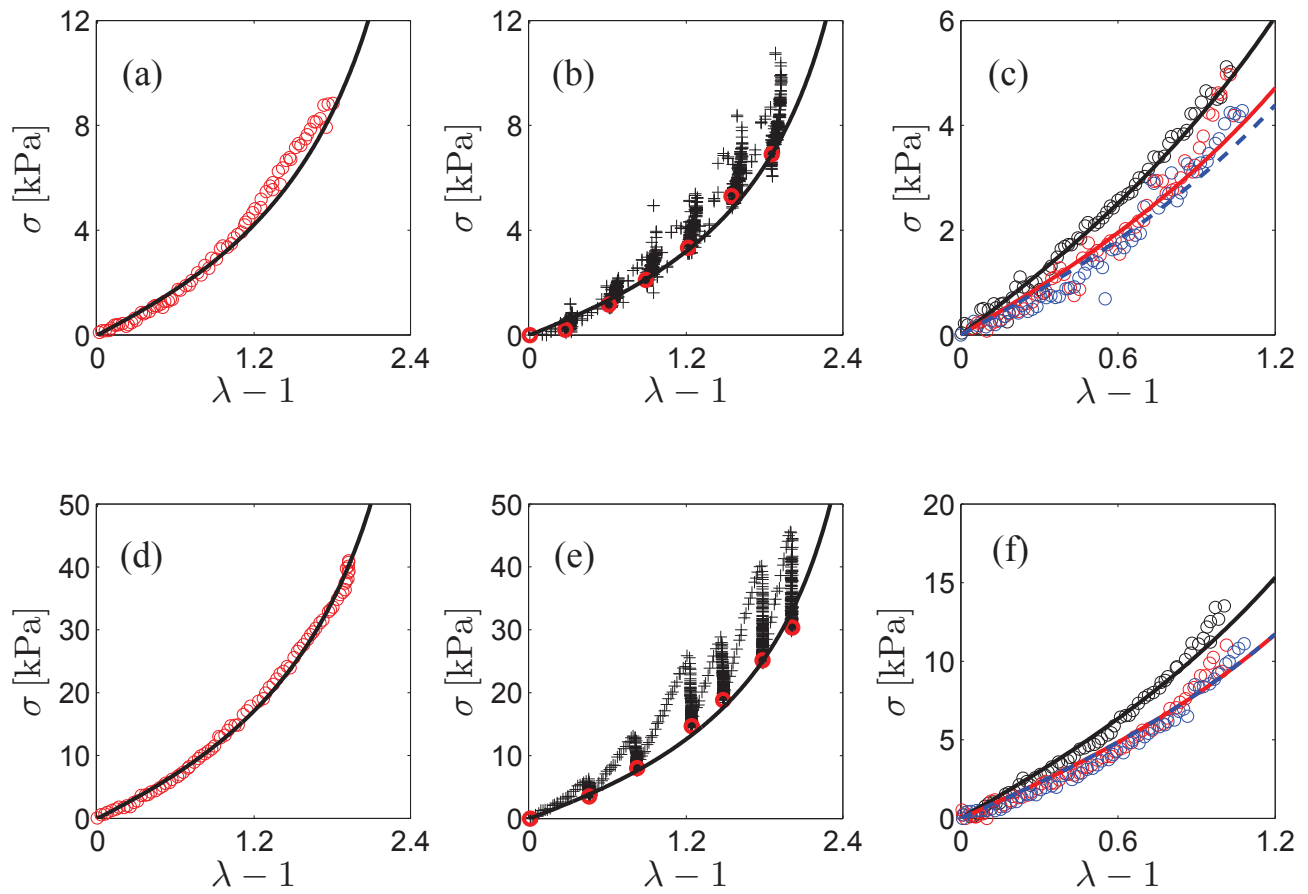

Figure 6: Experimentally measured mechanical response of the poly(NIPAAm-co-Py-PEGMA) nanocomposite hydrogel during uniaxial stretching (markers) and theoretical predictions using equation (1) (solid line):(a,d) single step loading; (b,e) stressrelaxation; and (c,f) cyclic loading, where black, red, and blue markers correspond to the first, second, and third loading cycle, respectively. Panels $(\mathrm{a}-\mathrm{c})$ refer to the $1.5 \mathrm{wt} . \%$ clay hydrogel and $(\mathrm{d}-\mathrm{f})$ to the $3.0 \mathrm{wt} . \%$ clay hydrogel.

(Lu et al., 2012; Wang et al., 2013a; Ye et al., 2015).

Similar absorption bands are observed for Py-PEGMA oligomers, with the addition of the absorption band at $848 \mathrm{~cm}^{-1}$ that is associated with stretching vibrations of aromatic rings in the pyrene molecule (Wu and Shi, 2005). After the esterification reaction between PEGMA and pyrene butyric acid (see the structures displayed in Figure 2), we observe an increase in the intensity of the peaks at 1248 and $1376 \mathrm{~cm}^{-1}$ as well as the formation of the shoulder at $1738 \mathrm{~cm}^{-1}$, which we attribute to the formation of the additional ester bond. Further, we observe an almost complete disappearance of the absorption band at $3460 \mathrm{~cm}^{-1}$ due to reaction of the hydroxyl group during esterification.

The infrared spectrum of poly(NIPAAm-co-Py-PEGMA) nanocomposite hydrogels is displayed in Figure 5(b) together with the spectrum of poly(NIPAAm) nanocomposite hydrogels synthesized following the procedure reported in Haraguchi and Takehisa (2002). Characteristic absorption bands of poly(NIPAAm) at $1638 \mathrm{~cm}^{-1}$ and $1527 \mathrm{~cm}^{-1}$ are associated with the $\mathrm{C}=\mathrm{O}$ and $\mathrm{C}-\mathrm{N}$ bending vibrations in the amide bond, respectively (Liu et al., 2012; Ye et al., 2015). The absorption band at $997 \mathrm{~cm}^{-1}$ is related to the absorption of the Laponite crosslinker (Liu et al., 2012). Copolymerization of NIPAAm with Py-PEGMA oligomers result in the appearance of the adsorption band at $1716 \mathrm{~cm}^{-1}$, associated to the ester bond in the Py-PEGMA (Ye et al., 2015), as well as in an increase of the absorption in the region encompassing the peak at $2868 \mathrm{~cm}^{-1}$.

\section{Mechanical response in large uniaxial deformations}

Large deformations of mechanochromic poly(NIPAAm-co-Py-PEGMA) nanocomposite hydrogels were studied under uniaxial tension, using the experimental setup previously described in Cellini et al. (2016). Samples containing 1.5 and $3 \mathrm{wt} \%$ clay were tested in three different conditions. A total of 30 samples were tested, with 15 samples for each concentration and five samples per condition. Since these hydrogels are characterized by a highly hydrophilic structure below the lower critical solution temperature (Luzon et al., 2010), samples were weighed prior to the experiments to ascertain that their mass would be comparable to 


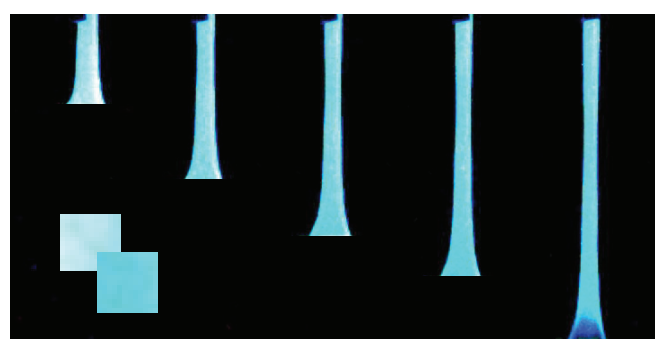

Figure 7: Stretching of a poly(NIPAAm-co-Py-PEGMA) nanocomposite hydrogel slab during uniaxial testing. Images recorded using a CMOS color camera (sample region close to the top clamp is covered by the testing equipment). In the inset, we display magnifications of pixels extracted from the leftmost and rightmost images, respectively.

the initial mass of the synthesized hydrogels. In addition, samples were kept hydrated until the beginning of each test.

In the first condition, slabs were stretched in a single step from $\lambda=1$ to $\lambda \approx 3$, where $\lambda$ is the uniaxial extension defined as the ratio between the deformed and the original length of the sample. Each experiment was approximately $40 \mathrm{~s}$ long, yielding a strain rate of approximately $0.05 \mathrm{~s}^{-1}$. During the test, we simultaneously measured the applied force, the uniaxial extension, and the fluorescence emission. In the second condition, samples were stretched in successive steps of approximately 0.3 from $\lambda=1$ to $\lambda \approx 3$ at the same deformation rate as the first condition, but holding the deformation constant for $60 \mathrm{~s}$ after each step. In the third condition, slabs were stretched from $\lambda=1$ to $\lambda \approx 2$ for three consecutive loading-unloading cycles, at the same rate of the first condition. Frames extracted from one of the videos recorded during the experiments are displayed in Figure 7.

To interpret the mechanical response of the gels, we adopt a modified form of the Arruda-Boyce model presented in Boyce and Arruda (2001), which accounts for the effect of solvent swelling on the mechanical properties of the gel network (Baek and Pence, 2011). The feasibility of utilizing such a constitutive theory for the study of hydrogels was demonstrated in Westbrook and Qi (2008), which focused on swollen PNIPAAm hydrogels. The approach extends the original work by Flory and Erman (Flory and Erman, 1982), by considering a non-Gaussian-based Arruda-Boyce eight chain model to capture large deformations. The model explicitly accounts for the so-called swelling ratio $v_{\mathrm{s}}$, which is defined as the ratio between the volume occupied by the gel network in the dry state and the volume of the swollen hydrogel.

In the case of uniaxial extension, the Cauchy stress is (Boyce and Arruda, 2001)

$$
\begin{aligned}
\sigma= & \frac{v_{\mathrm{s}}^{\frac{2}{3}} \mu}{6} \frac{\sqrt{N}}{\lambda_{\text {chain }}} \mathrm{L}^{-1}\left(\frac{\lambda_{\text {chain }}}{\sqrt{N} v_{\mathrm{s}}^{\frac{1}{3}}}\right)\left(\lambda^{2}-\frac{1}{\lambda}\right) \\
& +\frac{v_{\mathrm{s}} \mu}{8}\left(C_{1} \lambda^{2}-C_{2} \frac{1}{\lambda}\right)
\end{aligned}
$$

where $N$ is the number of statistical links in a chain between crosslinks, $\lambda_{\text {chain }}=\sqrt{\frac{1}{3}\left(\lambda^{2}+\frac{2}{\lambda}\right)}$ is the chain stretching in uniaxial extension, $\mu$ is a network parameter associated with the mechanical stiffness, and $\mathrm{L}^{-1}(x) \approx x \frac{3-x^{2}}{1-x^{2}}$ is the inverse Langevin function (Jedynak, 2014). The functions $C_{1}$ and $C_{2}$ are related to the geometric constraints imposed on the network and depend on the stretch $\lambda$. For uniaxial loading, these functions can be written as

$$
C_{1}=\left(1-\frac{1}{B_{1}+1}\right) \frac{1}{\lambda} \frac{\mathrm{d} B_{1}}{\mathrm{~d} \lambda}+\left(1-\frac{1}{D_{1}+1}\right) \frac{1}{\lambda} \frac{\mathrm{d} D_{1}}{\mathrm{~d} \lambda}
$$

with

$$
B_{1}=\alpha^{2} \frac{\left(v_{\mathrm{s}}^{-\frac{1}{3}} \lambda\right)^{2}-1}{\left(\left(v_{\mathrm{s}}^{-\frac{1}{3}} \lambda\right)^{2}+\alpha\right)^{2}}
$$


Table 1: Mean and standard deviation for mechanical properties measured during uniaxial loading for mechanochromic poly(NIPAAm-co-Py-PEGMA) nanocomposite hydrogels prepared with 1.5 and 3.0 wt.\% clay concentration.

\begin{tabular}{lllll}
\hline & $\mu(\mathrm{kPa})$ & $\mu_{\mathrm{eq}}(\mathrm{kPa})$ & $\mathrm{HR}_{1 \rightarrow 2}$ & $\mathrm{HR}_{2 \rightarrow 3}$ \\
\hline 1.5 wt. $\%$ & $3.19 \pm 0.82$ & $2.23 \pm 0.48$ & $0.18 \pm 0.10$ & $0.11 \pm 0.05$ \\
3.0 wt. $\%$ & $9.34 \pm 0.85$ & $7.06 \pm 0.80$ & $0.25 \pm 0.05$ & $0.10 \pm 0.05$ \\
\hline
\end{tabular}

$$
D_{1}=\frac{\left(v_{\mathrm{s}}^{-\frac{1}{3}} \lambda\right)^{2}}{\alpha} B_{1}
$$

and

$$
C_{2}=-2\left(1-\frac{1}{B_{2}+1}\right) \lambda^{2} \frac{\mathrm{d} B_{2}}{\mathrm{~d} \lambda}-2\left(1-\frac{1}{D_{2}+1}\right) \lambda^{2} \frac{\mathrm{d} D_{2}}{\mathrm{~d} \lambda}
$$

with

$$
\begin{gathered}
B_{2}=\alpha^{2} \frac{\left(\frac{v_{\mathrm{s}}^{-\frac{1}{3}}}{\sqrt{\lambda}}\right)^{2}-1}{\left(\left(\frac{v_{\mathrm{s}}^{-\frac{1}{3}}}{\sqrt{\lambda}}\right)^{2}+\alpha\right)^{2}} \\
D_{2}=\frac{1}{\alpha}\left(\frac{v_{\mathrm{s}}^{-\frac{1}{3}}}{\sqrt{\lambda}}\right)^{2} B_{2}
\end{gathered}
$$

Here, $\alpha$ is a model parameter in the original work by Flory and Erman (Flory and Erman, 1982), which encodes information about the constraints imposed on the polymer network.

Figure 6 displays representative experimental stress-strain curves for the three considered experimental conditions on hydrogel samples with both clay concentrations. The model in equation (1) is calibrated on the experimental dataset using $\mu$ as the fitting parameter (Cellini et al., 2014, 2016). Following Westbrook and Qi (2008), we assume $N=26.2$ and $\alpha=50$, which are reference values for crosslinked PNIPAAm networks, while we set $v_{\mathrm{s}}=0.1$ based on the initial hydrogel composition. Across all the experiments, we find that the model can accurately describe stress-strain data with a value of $\mu$ on the order of a few $\mathrm{kPa}$, in line with previous observations of this material system (Haraguchi and Takehisa, 2002; Okay and Oppermann, 2007).

By comparing panels $(\mathrm{a}-\mathrm{c})$ with $(\mathrm{d}-\mathrm{f})$, we observe a substantial dependence of the stiffness on the clay concentration, with the smaller concentration exhibiting a higher elastic compliance. Specifically, a twofold increase in clay concentration results into a threefold increase in the mechanical stiffness of the hydrogel. For example, a $100 \%$ stretch yields a stress between 4 and $5 \mathrm{kPa}$ for the $1.5 \mathrm{wt} . \%$ clay concentration and a value between 12 and $15 \mathrm{kPa}$ for the $3.0 \mathrm{wt} . \%$ clay concentration.

From panels (b) and (e), we observe stress relaxation at a constant deformation, indicating strain-rate dependence of the hydrogel. Specifically, during each deformation step of approximately $30 \%$, the stress rapidly rises as the sample stretches and then slowly relaxes toward equilibrium once the deformation is held constant. For each deformation step, the stress at equilibrium is determined by averaging the last 100 samples, totaling approximately 1 of the 60 s relaxation period. By comparing panels (a) with (b) and (d) with (e), we note a reduction in the mechanical stiffness along the equilibrium loading path as compared to the single-step loading condition. In particular, the values of the stress at equilibrium in panels (b) and (e) are slightly less than the corresponding stress levels in panels (a) and (d).

Panels (c) and (f) show the hydrogel stress-strain behavior over successive loading cycles. For both clay concentrations, the stiffness shows a modest decrease between the first and second loading cycles; no noticeable variation is observed between the second and third cycles. To quantify the degree of irreversibility of the mechanical response, we measure the so-called hysteresis ratio (HR), defined as the ratio between the difference of strain energy density between two successive cycles normalized by the strain energy in the first of the two cycles. The strain energy is computed by integrating the nominal stress, defined as $\sigma / \lambda$, 


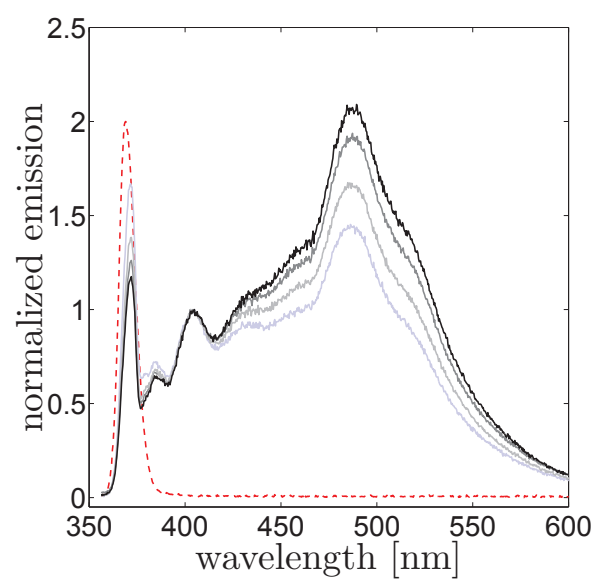

Figure 8: Emission spectra recorded at increasing strain levels during uniaxial stretching. The upper line (solid black) refers to the virgin state, bottom line (light gray) corresponds to the largest deformation $\lambda \approx 3$. Emission spectra are normalized with respect to the peak at $404 \mathrm{~nm}$. The dashed red line is the spectrum of the UV light utilized to excite the hydrogel fluorescence.

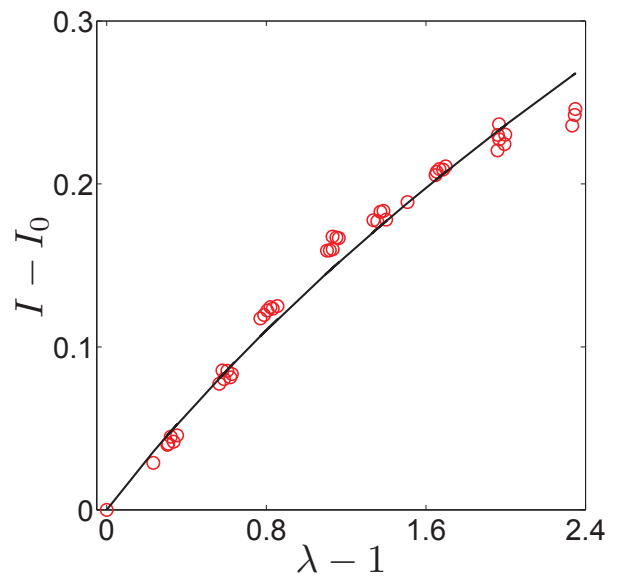

Figure 9: Difference between the emission ratios of the stretched poly(NIPAAm-co-Py-PEGMA) hydrogel and the virgin sample as a function of the uniaxial strain. Markers represent experimental data and the solid line the constitutive behavior identified using equation (4).

with respect to $\lambda^{2}$. In panel (c), the hysteresis ratios between the first and second cycles and between the second and third cycles are: $\mathrm{HR}_{1 \rightarrow 2}=0.16$ and $\mathrm{HR}_{2 \rightarrow 3}=0.08$, respectively. Similarly, for panel (f), we find $\mathrm{HR}_{1 \rightarrow 2}=0.17$ and $\mathrm{HR}_{2 \rightarrow 3}=0.05$.

Table 1 reports the average values of $\mu$ and the hysteresis ratios across all samples for both clay concentrations, along with their standard deviations. Consistent with the analysis of the representative data in Figure 6 , we observe a dependence of $\mu$ on the clay concentration, a reduction in $\mu$ due to stress relaxation, and minimal hysteretic behavior after the first loading cycle.

\section{Mechanochromic response}

To evaluate mechanochromism poly(NIPAAm-co-Py-PEGMA) nanocomposite hydrogels, fluorescence emission is recorded during tensile loading. Figure 8 displays representative emission spectra recorded at different strain levels ranging from $\lambda=1$ to $\lambda \approx 3$. Three peaks are identified at $372 \mathrm{~nm}, 388 \mathrm{~nm}$, and $404 \mathrm{~nm}$, which we associate with the emission of the pyrene structures in the monomer state. In addition,

\footnotetext{
${ }^{2}$ Alternatively, strain energy can be computed using Cauchy stress. In this case, the energy density is $\int_{\lambda_{0}}^{\lambda} \sigma \mathrm{d}\left(\ln \lambda^{\prime}\right)$.
} 

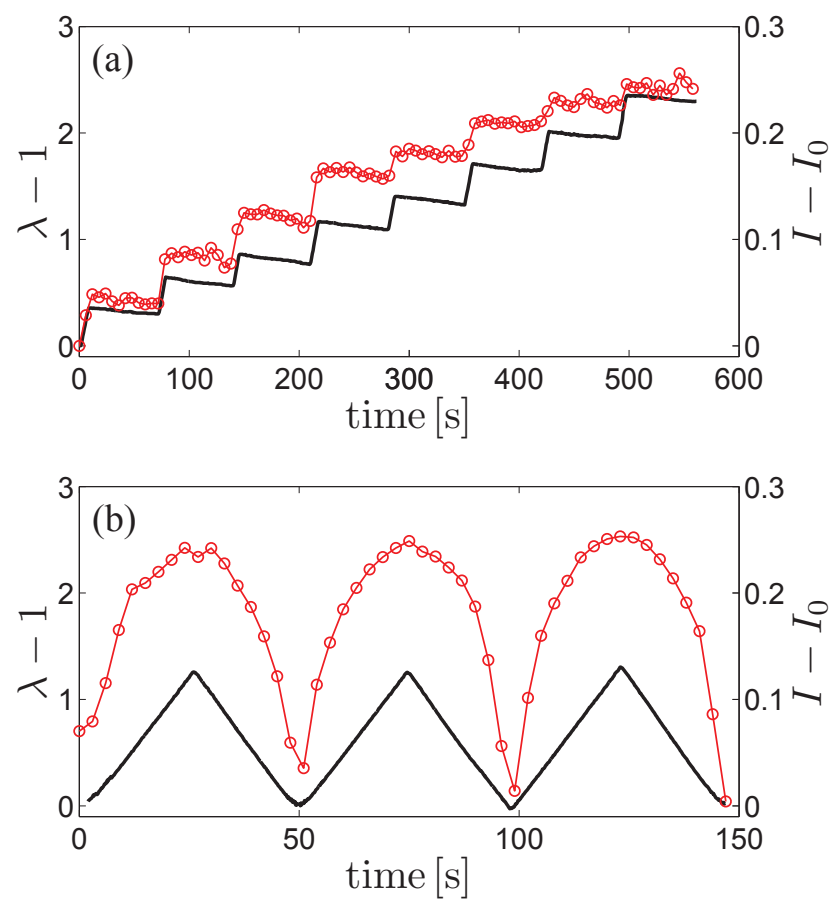

Figure 10: $\lambda-1$ (solid black line) and $I-I_{0}$ (markers) as functions of time during: (a) a stress relaxation and (b) a cyclic loading experiment.

the spectra show a broader peak approximately centered at $500 \mathrm{~nm}$, which is instead related to excimer emission of the dye.

Figure 8 also displays the emission of the UV light used to excite fluorescence. We note that the emission of the LED partially overlaps with the dye emission, especially with respect to the peak at the lowest wavelength of $372 \mathrm{~nm}$. While this may act as a confound in the attribution of the peak to the hydrogel mechanochromism, spectral data recorded using a fluorescence spectrophotometer with a single wavelength excitation at $350 \mathrm{~nm}$ confirm the presence of a peak at $372 \mathrm{~nm}$. We also report that a reduction of fluorescence emission due to photobleaching of pyrene conjugates incorporated in the hydrogel is not observed during the experiments.

In Figure 1, we propose a possible mechanism for the mechanochromic response of hydrogel networks. We hypothesize that mechanochromism is controlled by the progressive unfolding of the polymer chains, which, in turn, modulates intermolecular distances between pyrene molecules embedded in the nanocomposite hydrogel (Bao et al., 2013; Cellini et al., 2014; Crenshaw and Weder, 2006). More specifically, the increase of intermolecular distances between pyrene molecules is assumed to be related to the decrease of excimer emission with respect to monomer emission during stretching (Urban, 2011).

To gain further insight into hydrogel mechanochromism, we adapt the constitutive hypothesis presented in our previous work on mechanochromic elastomers (Cellini et al., 2016), which assumes a linear relationship between the change in emission and the stretch of the material surface (Lacarbonara, 2013). Specifically, we propose the following relationship for uniaxial stretching of mechanochromic hydrogel slabs:

$$
I-I_{0}=\beta(\sqrt{\lambda}-1)
$$

where $\beta$ is a constitutive parameter, $(\sqrt{\lambda}-1)$ is the stretch of the hydrogel surface as a function of $\lambda$, and $I$ and $I_{0}$ are the emission ratios in the deformed and virgin states, respectively. The emission ratio is defined as the ratio between the intensity of the emission in the monomer band, which is estimated from the intensity of the peak centered at $404 \mathrm{~nm}$, and the emission in the excimer band, estimated as the peak at $500 \mathrm{~nm}$. 
Table 2: Mean and standard deviation of $\beta$ and $I_{0}$ measured during uniaxial loading for mechanochromic poly(NIPAAm-coPy-PEGMA) nancomposite hydrogels prepared with 1.5 and $3.0 \mathrm{wt} . \%$ clay concentrations.

\begin{tabular}{lll}
\hline & $\beta$ & $I_{0}$ \\
\hline 1.5 wt. $\%$ & $0.24 \pm 0.06$ & $0.51 \pm 0.02$ \\
3.0 wt. $\%$ & $0.20 \pm 0.03$ & $0.53 \pm 0.03$ \\
\hline
\end{tabular}

Equation (4) posits that the change in the emission ratios is linearly proportional to the variation in the surface area of the sample during stretching. Moreover, since the lifetime of pyrene radiation is on the order of hundreds of nanoseconds (Birks et al., 1963), dye optical response is assumed to be almost instantaneous in our application. Thus, we do not account for the effective radiative lifetime of the pyrene structures in our analysis of mechanochromic response. Figure 9 displays $I-I_{0}$ as a function of the uniaxial stretch recorded during one of the experiments. The constitutive hypothesis underlying equation (4) successfully predicts the mechanochromic response in the whole range of imposed deformations.

Figure 10(a) and (b) contrasts the time traces of $I-I_{0}$ and $\lambda$ during a stress relaxation experiment and a cyclic loading experiment, respectively. Figure 10(a) demonstrates the strain dependence of the fluorescence emission. In agreement with our prior observations on mechanochromic elastomers (Cellini et al., 2014, 2016), the emission is controlled only by the deformation and does not vary as the stress relaxes towards equilibrium within each loading step. Thus, although it is tenable to hypothesize a viscoelastic behavior of the hydrogels, their mechanochromic response does not suggest strain-rate dependence.

Figure 10(b) demonstrates reversibility in the optical response, whereby we do not find variations in the fluorescence emission across the three loading cycles, despite the changes in the mechanical stiffness observed in Figure 6. Data presented in Figure 10(b) confirm the nonlinear dependence of the emission on the stretch, as predicted from the constitutive model in equation (4), which suggests a bell shape for the emission curve in response to linear loading and unloading curves.

\section{Pressure sensing}

To illustrate the possibility of differential pressure sensing using mechanochromic hydrogel membranes, inflation experiments are conducted on the setup described in Section 2. Membranes with $3 \mathrm{wt} \%$ clay and $1 \mathrm{wt} \%$ dye are installed on the setup and clamped down using three screws, see Figure 3. Pressure inside the pneumatic circuit is increased by incrementing approximately linearly the voltage supplied to the mini-pump. The membranes are inflated until failure, which occurs by burst and laceration of the hydrogel. Experiments have duration ranging between 80 and $120 \mathrm{~s}$, with the maximum height reached during inflation in the range of $28-32 \mathrm{~mm}$, corresponding to a strain at the apex of the membrane of approximately $1000 \%$.

Figure 11(a) and (b) display the mechanical and optical responses of the membrane during the test. Figure 11(a) illustrates the pressure in the pneumatic circuit and the membrane height during inflation. In the first 5 seconds of the test, the pressure rapidly increases from 0 to $0.5 \mathrm{kPa}$. In the interval between 5 and 45 seconds, the pressure increases to $1 \mathrm{kPa}$ at a slower rate. In the final part of the test, the pressure robustly increases to $2.2 \mathrm{kPa}$, reaching a maximum before bursting. Due to the differential air pressure between the circuit and the environment, the membrane progressively inflates during the experiment. During the first $5 \mathrm{~s}$, the height of the membrane increases from 0 to $3.5 \mathrm{~mm}$. This initial ramp is followed by a slower expansion with the membrane's height reaching $7 \mathrm{~mm}$ after $45 \mathrm{~s}$ and a final expansion up to a height of approximately $30 \mathrm{~mm}$ before rupture.

Figure 11(b) shows the mechanochromic response of the membrane during inflation. Similar to tensile experiments, we observe a progressive increase of the emission ratio during deformation. As expected, the change of optical properties correlates with the stretching of the hydrogel, following the change in the height. To estimate the resolution of pressure sensing, we define the pressure sensitivity of the mechanochromic membrane as $K=\frac{1}{I_{0}} \frac{\Delta I}{\Delta p}$, where $\Delta I$ and $\Delta p$ are the total variation in the emission ratio and pressure, respectively, and $h$ is the nominal thickness. By averaging four independent experiments on virgin samples, we compute a sensitivity of $K=0.28 \pm 0.09 \mathrm{kPa}^{-1} \mathrm{~mm}$. Despite the relative simplicity of the experimental configuration, this value is only one order of magnitude lower than sensitivities obtained for solid-state 

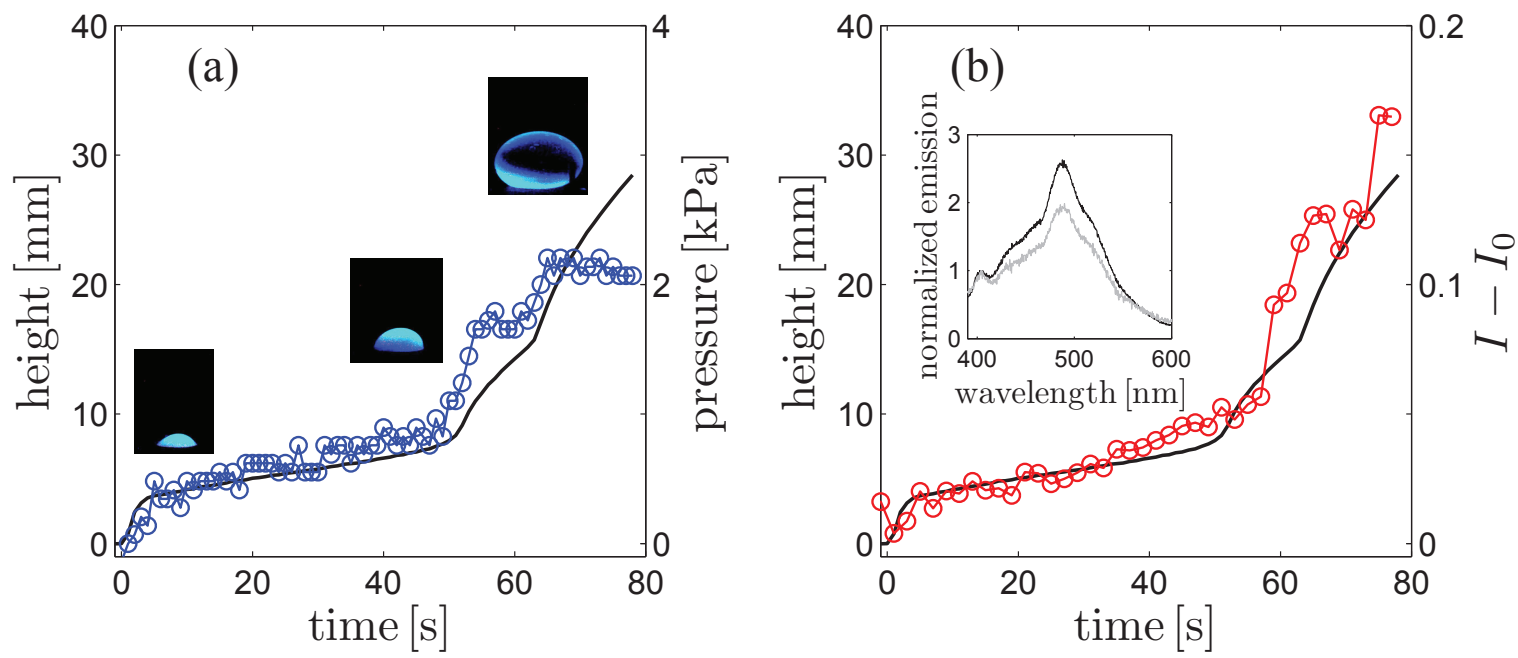

Figure 11: (a) Membrane height during inflation (solid line) and internal pressure (blue circles) as a function of time. (b) Membrane height during inflation and emission ratio (red dots) as a function of time. Insets in (a) show membrane inflation at 4,52 , and $78 \mathrm{~s}$. Inset in (b) shows spectra recorded at the beginning and at the end of the inflation experiment.

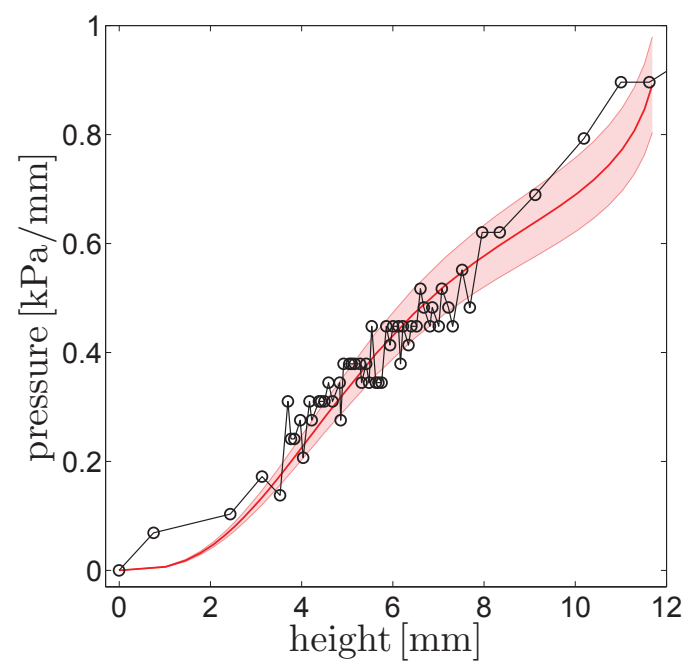

Figure 12: Experimental data on the pressure as a function of the membrane height (black circles) and theoretical predictions from the model in Cellini et al. (2016), adapting constitutive equation (1). The shaded region represents theoretical predictions obtained by varying the parameter $\mu$ within one standard deviation from Table 1 .

devices based on conductive polymers (Choong et al., 2014; Shao et al., 2014) and high sensitivity Fiber Bragg Grating (Song et al., 2014; Zhang et al., 2001).

To compare results from the pressure sensing experiment with uniaxial tests, the parameters identified from uniaxial stretching are used to predict the mechanical response of the membrane by adapting the analytical model discussed in our previous work (Cellini et al., 2016). Specifically, the constitutive hypothesis used in Cellini et al. (2016) is modified by accounting for equation (1). Since $v_{\mathrm{s}}=0.1$, the first term in equation (1) dominates the constitutive behavior of the highly swollen membrane, which is equivalent to the unconstrained network (Westbrook and Qi, 2008). As a result, we discard the second term in (1) for the numerical integration of the deformation as a function of the pressure. We focus our analysis on the initial part of the pressure loading curve, where values of strain rate $\left(0.06 \mathrm{~s}^{-1}\right)$ and membrane strains $(\lambda \approx 3)$ are comparable with values obtained during uniaxial experiments. Experimental values of the pressure as a 
function of the membrane height are displayed in Figure 12, together with theoretical predictions determined using values of the parameter $\mu$ in Table 1 . The model predicts correctly the pressure range in the circuit as a function of the membrane deformation.

\section{Conclusions}

In this paper, we presented results for the synthesis and optomechanical characterization of mechanochromic poly(NIPAAm-co-Py-PEGMA) nanocomposite hydrogels. To enable mechanochromism, we ad-hoc synthesized pyrene terminated polyethylene glycol methacrylate oligomers (Py-PEGMA), which we propose as active mechanochromic chromofores in the nanocomposite hydrogels.

Fluorescence spectra of Py-PEGMA evidenced the classical landscape of emission of pyrene derivatives, with an increase in the relative intensity of excimer to momomer emissions for increasing concentrations of Py-PEGMA. On the other hand, no relative intensity variations were observed across the different UV-Vis absorption bands. ATR-FTIR and 1D proton NMR experiments were conducted to characterize Py-PEGMA oligomers. Analysis of the spectra indicates: i) the presence of aromatic pyrene molecules in the synthesized compound; ii) the formation of an additional ester bond due to the esterification reaction between the carboxylic acid of the PyBA and the hydroxyl group of the methoxy terminated PEGMA; and iii) the consequent disappearance of characteristic peaks associated with carboxylic acid of PyBA and hydroxyl group of PEGMA after esterification.

Synthesis of pyrene functionalized nanocomposite hydrogels was carried by adapting the formulation presented in Haraguchi and Takehisa (2002). Optomechanical analysis of the hydrogels was performed on a dedicated experimental setup allowing simultaneous acquisition of force, deformation, and fluorescence emission. Mechanical data collected for hydrogels containing $1.5 \mathrm{wt} \%$ and $3 \mathrm{wt} \%$ clay and $1 \mathrm{wt} \%$ dye were studied using a modified form of the Arruda-Boyce model, which accounts for the effect of swelling. A threefold increase of the stiffness was observed by increasing the clay content from 1.5 to 3 wt\%. Stress relaxation and repeated loading experiments suggested the presence of non-secondary viscoelastic phenomena and non-reversible hysteresis between the first and second loading cycles.

To investigate mechanochromism, fluorescence emission was collected during tensile experiments. During uniaxial stretching, we observed a progressive increase of the emission ratio, which we associated with the reorganization of dyes within the structure. Experiments confirmed that the mechanochromic response is repeatable. No significant contribution from time-dependent relaxation phenomena was detected in the optical response of the hydrogels, which is in line with our previous observations on mechanochromic elastomers (Cellini et al., 2016). To analyze emission data, we adapted the constitutive hypothesis proposed in Cellini et al. (2016), and found it to successfully describe the functional relation between the emission ratio and the stretching of the hydrogel, offering direct evidence that the emission linearly correlates with the surface stretch.

Finally, differential pressure sensing was demonstrated using mechanochromic hydrogel membranes. Hydrogel membranes were installed on a pneumatic circuit and progressively loaded by increasing the internal pressure. Pressure, membrane height, and emission were recorded over time during the test. Experimental results demonstrated that mechanochromic hydrogel membranes have pressure sensitivity of approximately $0.28 \mathrm{kPa}^{-1} \mathrm{~mm}$, which is in line with more established, state-of-the-art technologies (Choong et al., 2014; Shao et al., 2014; Song et al., 2014; Zhang et al., 2001).

We envision the application of mechanochromic nanocomposite hydrogels in the design of new force and pressure sensors in solid mechanics and fluid dynamics. In particular, the possibility of sensing forces in the order of a few millinewtons and pressures of a few hundred Pascals opens avenues to the design of sensors for biological fluid mechanics, where the ability of accurately resolving flow kinetics is of fundamental importance.

\section{Aknowledgments}

This research was conducted with the support of the National Science Foundation through Grant No. CBET-1332204. The authors also acknowledge support from the Office of Naval Research through Grant 
No. N00014-10-1-0988, which has allowed the acquisition of equipment used in this study. The authors want to thank Dr. Avi Ulman and the Institute for Engineered Interfaces (IEI) at the New York University Tandon School of Engineering for granting access to the laboratories. The authors acknowledge the Graduate Programs Office at New York University Abu Dhabi for partially supporting Filippo Cellini's visit, during which part of the research presented herein was conducted. Finally, the authors thank Mr. Adel Shams for his careful review of the manuscript.

\section{Appendix A. Proton NMR spectrum and mass analysis of Py-PEGMA oligomers}

The 1D proton NMR spectrum of Py-PEGMA is recorded using a Bruker AV III $500 \mathrm{MHz}$ NMR instrument. Py-PEGMA oligomers are synthesized following the procedure outlined in Section 2 and further purified through column chromatography, using silica gel as the stationary phase and a 2.5:100 solution of Methanol:Dichlorometane as the eluent (Lapienis and Penczek, 2001). After purification, Py-PEGMA oligomers are recovered from solution by evaporation under reduced pressure, and NMR samples are prepared by redissolving the Py-PEGMA in deuterated dimethyl sulfoxide (DMSO-d6). Figures A.13(a) and (b) display the 1D proton NMR spectrum of Py-PEGMA together with the spectrum of unmodified PEGMA oligomers, which are analyzed as received without further purification. Peaks pertaining to residual solvent traces are identified through comparison with reference NMR data (Fulmer et al., 2010). In particular, we detect traces of $\mathrm{H}$ protons of DMSO with chemical shift $2.50 \mathrm{ppm}, \mathrm{H}_{2} \mathrm{O}$ with chemical shift $3.34 \mathrm{ppm}$, and DCM with chemical shift $5.76 \mathrm{ppm}$.

Chemical shifts associated with proton resonances of PEGMA and Py-PEGMA are identified in Figure A.13 using numbers 1 to 7 (chemical structures are displayed in Figure 2). Peak 1 at 1.88 ppm is associated with the resonance of the methyl group $\mathrm{CH}_{3}-$ of the methacrylate (Wang et al., 2013b; Zhou et al., 2011). In addition, resonances of protons in the methene group $\mathrm{CH}_{2}=$ of the methacrylate are found at chemical shifts $5.69 \mathrm{ppm}$ and $6.04 \mathrm{ppm}$ corresponding to peaks 6a and 6b, respectively (Wang et al., 2013b; Zhou et al., 2011). Peaks in region 3, encompassing the broad range of chemical shifts between 3.4 and $3.7 \mathrm{ppm}$, are resonances pertaining to the polyethylene glycol repeated units $\left(-\mathrm{CH}_{2}-\mathrm{CH}_{2}-\mathrm{O}-\right.$ ) (Dust et al., 1990; Wang et al., 2013b; Zhou et al., 2011). Peaks in the region 4 at $4.21 \mathrm{ppm}$ are associated with chemical shifts of protons of the methene group in the ester $-\mathrm{CH}_{2}-\mathrm{O}-\mathrm{C}=\mathrm{O}$ (Wang et al., 2013b; Zhou et al., 2011). Peaks in region 5 at $4.57 \mathrm{ppm}$ are related to resonance of the proton in the the terminal hydroxyl group $\mathrm{OH}-$ (Dust et al., 1990).

Peaks 2 and 7 are specific of the structure of Py-PEGMA oligomers and are not found in the structure of pure PEGMA. In particular, the peaks in region 7 ranging from 7.90 to $8.45 \mathrm{ppm}$ are attributed to the resonance of protons in the aromatic rings of the pyrene molecule (Rossi et al., 2009). Further, we attribute peaks in region 2 at $2.02 \mathrm{ppm}$ to resonances of protons in the methylene chain connecting the terminal pyrene to the polyethylene glycol repeated units (Pham et al., 1983). By comparing the NMR spectra of PEGMA and Py-PEGMA oligomers, we observe a relative increase in the intensity of peaks in the region 4 at $4.21 \mathrm{ppm}$, associated to the protons in the ester bond. At the same time, we note a consistent reduction of intensity of the peaks in the region 5 at $4.57 \mathrm{ppm}$ associated with the terminal hydroxyl group. Such variations are attributed to the formation of the additional ester bond during reaction of carboxylic acid of PyBA with the terminal hydroxyl group of the PEGMA, with reference to chemical structures depicted in Figure 2. To further confirm our observations and offer some proof for the formation of the ester bond, we report that the characteristic resonance of the hydroxyl group of the carboxylic acid in the PyBA molecule located at $12.20 \mathrm{ppm}$ is not observed in spectrum of the Py-PEGMA oligomers (AIST, 2016).

The molecular weight of Py-PEGMA oligomers is measured using an Agilent 1100 Series Capillary LCMSD Trap XCT Spectrometer. After purification through column chromatography in silica gel (2.5:100 solution of Methanol:Dichlorometane as eluent), Py-PEGMA oligomers are dried under reduced pressure and redissolved in acetonitrile $(0.5 \mathrm{mg} / \mathrm{ml})$. Result of the mass analysis shows the presence of a distribution of molecular weights centered at $617.4 \mathrm{~m} / \mathrm{z}$, which is close to the expected average molecular weight of Py-PEGMA oligomers $(620.72 \mathrm{~m} / \mathrm{z}$, with reference to the chemical structure displayed in Figure 2). 

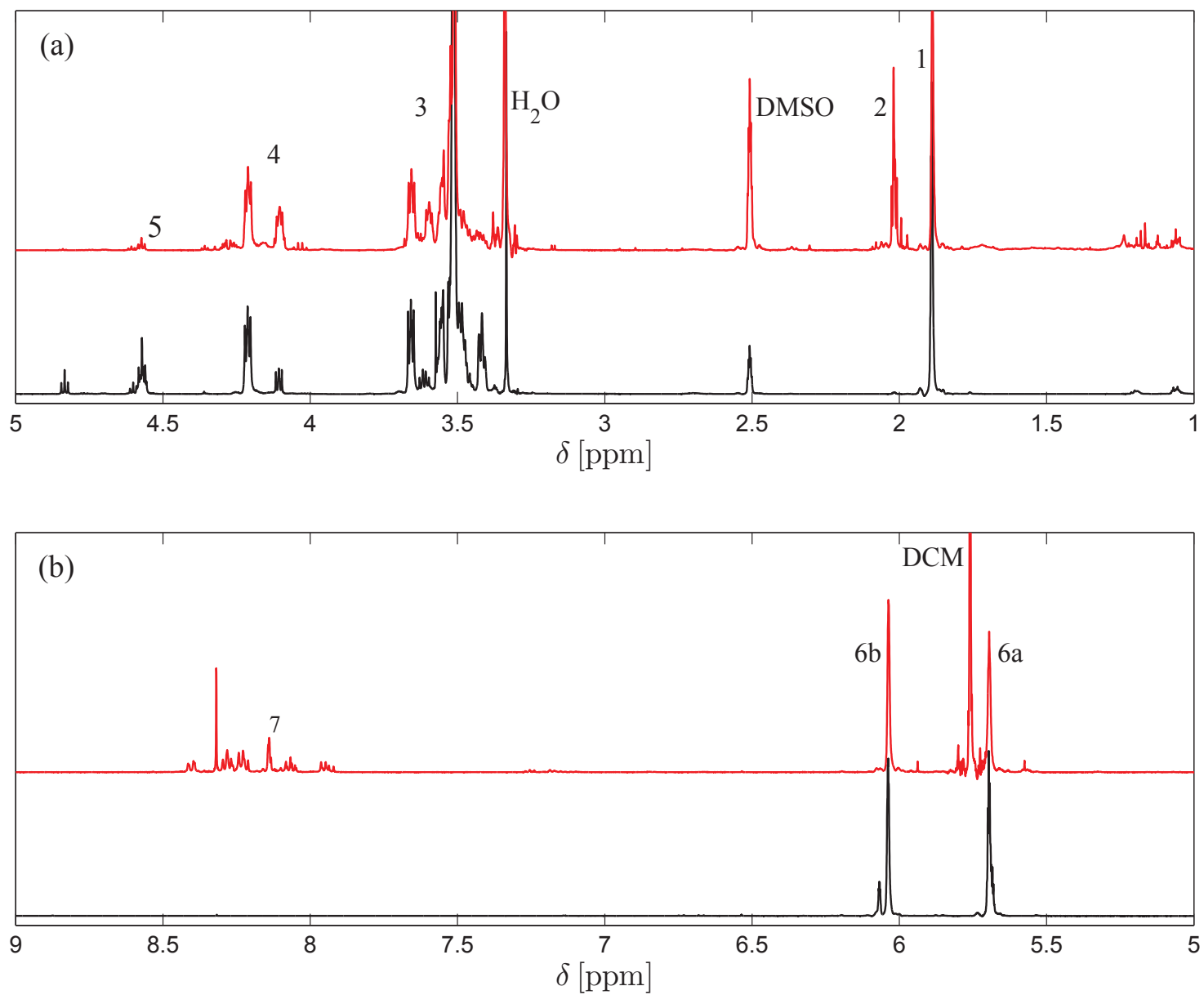

Figure A.13: (a) Chemical shifts in the region 1-5 ppm and (b) in the region $5-9$ ppm of 1 D proton NMR spectra (500 MHz) of PEGMA (black line) and Py-PEGMA (red line) oligomers in DMSO-d6.

\section{References}

AIST, 2016. Spectral Database of Organic Compounds: http://riodb01.ibase.aist.go.jp/sdbs.

Amano, Y., Hidema, R., Gong, J., Furukawa, H., 2012. Creation of shape-memory gels with inter-crosslinking network structure. Chemistry Letters 41, 1029-1031.

Baek, S., Pence, T.J., 2011. Inhomogeneous deformation of elastomer gels in equilibrium under saturated and unsaturated conditions. Journal of the Mechanics and Physics of Solids 59, $561-582$.

Bains, G., Patel, A.B., Narayanaswami, V., 2011. Pyrene: A probe to study protein conformation and conformational changes. Molecules 16, 7909 .

Bao, S., Li, J., Lee, K.I., Shao, S., Hao, J., Fei, B., Xin, J.H., 2013. Reversible mechanochromism of a luminescent elastomer. Applied Materials and Interfaces 5, 4625-4631.

Birks, J.B., Christophorou, L.G., 1963. Excimer fluorescence spectra of pyrene derivatives. Spectrochimica Acta 19, $401-410$.

Birks, J.B., Dyson, D.J., Munro, I.H., 1963. Excimer fluorescence. ii. lifetime studies of pyrene solutions. Proceedings of the Royal Society of London A: Mathematical, Physical and Engineering Sciences 275, 575-588.

Boyce, M.C., Arruda, E.M., 2001. Swelling and mechanical stretching of elastomeric materials. Mathematics and Mechanics of Solids 6, 641-659.

Buenger, D., Topuz, F., Groll, J., 2012. Hydrogels in sensing applications. Progress in Polymer Science 37, 1678 - 1719.

Cellini, F., Khapli, S., Peterson, S.D., Porfiri, M., 2014. Mechanochromic polyurethane strain sensor. Applied Physics Letters $105,061907$.

Cellini, F., Zhou, L., Khapli, S., Peterson, S.D., Porfiri, M., 2016. Large deformations and fluorescence response of mechanochromic polyurethane sensors. Mechanics of Materials 93, 145-162.

Chang, C.W., van Spreeuwel, A., Zhang, C., Varghese, S., 2010. Peg/clay nanocomposite hydrogel: a mechanically robust tissue engineering scaffold. Soft Matter 6, 5157-5164. 
Chen, Z., Tan, X., Will, A., Ziel, C., 2007. A dynamic model for ionic polymer-metal composite sensors. Smart Materials and Structures 16, 1477-1488.

Chi, Z., Zhang, X., Xu, B., Zhou, X., Ma, C., Zhang, Y., Liua, S., Xu, J., 2012. Recent advances in organic mechanofluorochromic materials. Chemical Society Reviews 41, 3878-3896.

Choong, C.L., Shim, M.B., Lee, B.S., Jeon, S., Ko, D.S., Kang, T.H., Bae, J., Lee, S.H., Byun, K.E., Im, J., Jeong, Y.J., Park, C.E., Park, J.J., Chung, U.I., 2014. Highly stretchable resistive pressure sensors using a conductive elastomeric composite on a micropyramid array. Advanced Materials 26, 3451-3458.

Ciardelli, F., Ruggeri, G., Pucci, A., 2013. Dye-containing polymers: methods for preparation of mechanochromic materials. Chemical Society Reviews 42, 857-870.

Crenshaw, B.R., Weder, C., 2006. Self-assessing photoluminescent polyurethanes. Macromolecules 39, $9581-9589$.

Debashish, R., Cambre, J.N., Sumerlin, B.S., 2010. Future perspectives and recent advances in stimuli-responsive materials. Progress in Polymer Science 35, 278-301.

Deligkaris, K., Tadele, T.S., Olthuis, W., van den Berg, A., 2010. Hydrogel-based devices for biomedical applications. Sensors and Actuators B: Chemical 147, $765-774$.

Donati, F., Pucci, A., Cappelli, C., Mennucci, B., Ruggeri, G., 2008. Modulation of the optical response of polyethylene films containing luminescent perylene chromophores. Journal of Physical Chemistry B 112, 3668-3679.

Duhamel, J., 2012. Internal dynamics of dendritic molecules probed by pyrene excimer formation. Polymers $4,211$.

Dust, J.M., Fang, Z.H., Harris, J.M., 1990. Proton nmr characterization of poly(ethylene glycols) and derivatives. Macromolecules 23, 3742-3746.

Ferrari, M., Bashir, R., Wereley, S.T. (Eds.), 2007. BioMEMS and Biomedical Nanotechnology. Volume IV: Biomolecular Sensing, Processing and Analysis. Springer.

Flory, P.J., Erman, B., 1982. Theory of elasticity of polymer networks. 3. Macromolecules 15, 800-806.

Fulmer, G.R., Miller, A.J.M., Sherden, N.H., Gottlieb, H.E., Nudelman, A., Stoltz, B.M., Bercaw, J.E., Goldberg, K.I., 2010. $\mathrm{Nmr}$ chemical shifts of trace impurities: Common laboratory solvents, organics, and gases in deuterated solvents relevant to the organometallic chemist. Organometallics 29, 2176-2179.

Gossweiler, G.R., Hewage, G.B., Soriano, G., Wang, Q., Welshofer, G.W., Zhao, X., Craig, S.L., 2014. Mechanochemical activation of covalent bonds in polymers with full and repeatable macroscopic shape recovery. ACS Macro Letters 3, 216219.

Haehnel, A.P., Sagara, Y., Simon, Y.C., Weder, C., 2015. Mechanochemistry in polymers with supramolecular mechanophores, in: Topics in Current Chemistry. Springer Berlin Heidelberg, pp. 1-31.

Haraguchi, K., 2007. Nanocomposite hydrogels. Current Opinion in Solid State and Materials Science 11, 47-54.

Haraguchi, K., Li, H., Matsuda, K., Takehisa, T., Elliot, E., 2005. Mechanism of forming organic/inorganic network structures during in-situ free-radical polymerization in pnipa-clay nanocomposite hydrogels. Macromolecules 38, 3482-3490.

Haraguchi, K., Takehisa, T., 2002. Nanocomposite hydrogels: A unique organic-inorganic network structure with extraordinary mechanical, optical, and swelling/de-swelling properties. Advanced Materials 14, 1120-1124.

Hu, X., Wang, T., Xiong, L., Wang, C., Liu, X., Tong, Z., 2010. Preferential adsorption of poly(ethylene glycol) on hectorite clay and effects on poly(n-isopropylacrylamide)/hectorite nanocomposite hydrogels. Langmuir 26, 4233-4238.

Imato, K., Kanehara, T., Ohishi, T., Nishihara, M., Yajima, H., Ito, M., Takahara, A., Otsuka, H., 2015. Mechanochromic dynamic covalent elastomers: Quantitative stress evaluation and autonomous recovery. ACS Macro Letters 4, $1307-1311$.

Jedynak, R., 2014. Approximation of the inverse langevin function revisited. Rheologica Acta , 1-11.

Jo, C., Pugal, D., Oh, I.K., Kim, K.J., Asaka, K., 2013. Recent advances in ionic polymer-metal composite actuators and their modeling and applications. Progress in Polymer Science 38, 1037-1066.

Kunzelman, J., Chung, T., Mather, P.T., Weder, C., 2008a. Shape memory polymers with built-in threshold temperature sensors. Journal of Materials Chemistry 18, 1082-1086.

Kunzelman, J., Crenshaw, B.R., Kinami, M., Weder, C., 2006. Self-assembly and dispersion of chromogenic molecules: A versatile and general approach for self-assessing polymers. Macromolecular Rapid Communications 27, $1981-1987$.

Kunzelman, J., Kinami, M., Crenshaw, B., Protasiewicz, J., Weder, C., 2008b. Oligo (p-phenylene vinylene) s as a "new" class of piezochromic fluorophores. Advanced Materials 20, 119-122.

Lacarbonara, W., 2013. Nonlinear Structural Mechanics. Springer.

Lapienis, G., Penczek, S., 2001. Preparation of monomethyl ethers of poly(ethylene glycol)s free of the poly(ethylene glycol). Journal of Bioactive and Compatible Polymers 16, 206-220.

Li, W., Huang, D., Wang, J., Shen, W., Chen, L., Yang, S., Zhu, M., Tang, B., Liang, G., Xu, Z., 2015. A novel stimuliresponsive fluorescent elastomer based on an AIE mechanism. Polymer Chemistry 6, 8194-8202.

Liu, D., Wang, T., Liu, X., Tong, Z., 2012. Accelerated cell sheet detachment by copolymerizing hydrophilic peg side chains into pnipam nanocomposite hydrogels. Biomedical Materials 7, 055008.

Lowe, C., Weder, C., 2002. Oligo(p-phenylene vinylene) excimers as molecular probes: Deformation-induced color changes in photoluminescent polymer blends. Advanced Materials 14, 1626-1629.

Lu, C., Bhatt, L.R., Jun, H.Y., Park, S.H., Chai, K.Y., 2012. Carboxyl-polyethylene glycol-phosphoric acid: a ligand for highly stabilized iron oxide nanoparticles. Journal of Materials Chemistry 22, 19806-19811.

Luzon, M., Boyer, C., Peinado, C., Corrales, T., Whittaker, M., Tao, L., Davis, T.P., 2010. Water-soluble, thermoresponsive, hyperbranched copolymers based on peg-methacrylates: Synthesis, characterization, and lcst behavior. Journal of Polymer Science Part A: Polymer Chemistry 48, 2783-2792.

Matzeu, G., Florea, L., Diamond, D., 2015. Advances in wearable chemical sensor design for monitoring biological fluids. Sensors and Actuators B: Chemical 211, $403-418$

Okay, O., Oppermann, W., 2007. Polyacrylamideclay nanocomposite hydrogels: rheological and light scattering characterization. 
Macromolecules 40, 3378-3387.

Pham, Q.T., Petiaud, R., Waton, H., 1983. Proton and Carbon NMR Spectra of Polymers. John Wiley \& Sons.

Pucci, A., Bertoldo, M., Bronco, S., 2005. Luminescent bis(benzoxazolyl)stilbene as a molecular probe for poly(propylene) film deformation. Macromolecular Rapid Communications 26, 1043-1048.

Pucci, A., Bizzarri, R., Ruggeri, G., 2011. Polymer composites with smart optical properties. Soft Matter 7, 3689-3700.

Roberts, D.R.T., Patel, M., Murphy, J.J., Holder, S.J., 2012. Optical response to stress in pyrene labelled polydimethylsiloxane elastomers: Monitoring strain in 1d and 2d. Sensors and Actuators B: Chemical 162, $43-56$.

Rossi, N.A.A., Duplock, E.J., Meegan, J., Roberts, D.R.T., Murphy, J.J., Patel, M., Holder, S.J., 2009. Synthesis and characterisation of pyrene-labelled polydimethylsiloxane networks: towards the in situdetection of strain in silicone elastomers. Journal of Materials Chemistry 19, 7674-7686.

Schexnailder, P., Schmidt, G., 2009. Nanocomposite polymer hydrogels. Colloid and Polymer Science 287, 1-11.

Shahinpoor, M. (Ed.), 2016. Ionic Polymer Metal Composites (IPMCs). RSC Smart Materials, The Royal Society of Chemistry.

Shao, Q., Niu, Z., Hirtz, M., Jiang, L., Liu, Y., Wang, Z., Chen, X., 2014. High-performance and tailorable pressure sensor based on ultrathin conductive polymer film. Small 10, 1466-1472.

Song, F., Xie, A.J., Seo, S.W., 2014. Elastomeric polymer resonant waveguide grating based pressure sensor. Journal of Optics 16,065702 .

Urban, M.W. (Ed.), 2011. Handbook of Stimuli-Responsive Materials,. Wiley-V.C.H. Verlag, Weinheim, Germany.

Wang, H., Shen, J., Cao, G., Gai, Z., Hong, K., Debata, P.R., Banerjee, P., Zhou, S., 2013a. Multifunctional peg encapsulated fe3o4@silver hybrid nanoparticles: antibacterial activity, cell imaging and combined photothermo/chemo-therapy. Journal of Materials Chemistry B 1, 6225-6234.

Wang, J.H., Altukhov, O., Cheng, C.C., Chang, F.C., Kuo, S.W., 2013b. Supramolecular structures of uracil-functionalized peg with multi-diamidopyridine poss through complementary hydrogen bonding interactions. Soft Matter 9, 5196-5206.

Westbrook, K.K., Qi, H.J., 2008. Actuator designs using environmentally responsive hydrogels. Journal of Intelligent Material Systems and Structures 19, 597-607.

Wu, X., Shi, G., 2005. Synthesis of a carboxyl-containing conducting oligomer and non-covalent sidewall functionalization of single-walled carbon nanotubes. Journal of Materials Chemistry 15, 1833-1837.

Yao, C., Kraatz, H.B., Steer, R.P., 2005. Photophysics of pyrene-labelled compounds of biophysical interest. Photochemical and Photobiological Sciences 4, 191-199.

Ye, Y., Huang, J., Wang, X., 2015. Fabrication of a self-cleaning surface via the thermosensitive copolymer brush of p(nipaampegma). ACS Applied Materials \& Interfaces 7, 22128-22136.

Zhang, Y., Feng, D., Liu, Z., Guo, Z., Dong, X., Chiang, K., Chu, B.C.B., 2001. High-sensitivity pressure sensor using a shielded polymer-coated fiber bragg grating. Photonics Technology Letters, IEEE 13, 618-619.

Zhou, G., Ma, C., Zhang, G., 2011. Synthesis of polyurethane-g-poly(ethylene glycol) copolymers by macroiniferter and their protein resistance. Polymer Chemistry 2, 1409-1414. 


\section{Biographies}

Filippo Cellini is a Ph.D. Candidate in the Department of Mechanical and Aerospace Engineering at the New York University Tandon School of Engineering. He received a B.S. degree in Aerospace Engineering and a M.Sc. degree in Aeronautical Engineering from Sapienza University of Rome. His current research interests include mechanochromic composites and smart materials and structures for sensing applications in fluids.

Leanne Block is an Undergraduate Student in Mechanical Engineering at Brown University. During summer 2015, she contributed to this research working as an Undergraduate Research Assistant at the New York University Tandon School of Engineering.

Jie Li is an Undergraduate Student in Physics at Skidmore College. During summer 2015, he contributed to this research working as an Undergraduate Research Assistant at the New York University Tandon School of Engineering

Sachin Khapli is currently an Assistant Professor in the Division of Engineering, New York University Abu Dhabi, UAE. He received his B.S. degree from IIT Kharagpur in 2000 and Ph.D. from Rice University in 2007, respectively. His research interests include the fabrication of nanostructured materials using supercritical fluid technology, development of hierarchically porous scaffolds for bone-tissue engineering, and development of smart hydrogels for biosensing.

Sean D. Peterson is an Associate Professor in the Department of Mechanical and Mechatronics Engineering at the University of Waterloo. He received his M.Sc. and Ph.D. degrees from Purdue University. His active research areas include fluid-structure interactions, vortex dynamics, biological fluid flows, and non-intrusive diagnostic techniques for fluid flows.

Maurizio Porfiri is a Professor in the Department of Mechanical and Aerospace Engineering at New York University. He received M.Sc. and Ph.D. degrees in Engineering Mechanics from Virginia Tech; a "Laurea" in Electrical Engineering and a Ph.D. in Theoretical and Applied Mechanics from Sapienza University of Rome and the University of Toulon. He is engaged in conducting and supervising research on dynamical systems theory, multiphysics modeling, and underwater robotics. 